\title{
Vaginal Mixed Epithelial and Mesenchymal Neoplasm
}

National Cancer Institute

\section{Source}

National Cancer Institute. Vaginal Mixed Epithelial and Mesenchymal Neoplasm. NCI

Thesaurus. Code C40274.

A benign or malignant neoplasm that arises from the vagina and is characterized by the presence of epithelial and mesenchymal elements. This category includes benign mixed tumor, adenosarcoma, carcinosarcoma, and malignant mixed tumor resembling synovial sarcoma. 